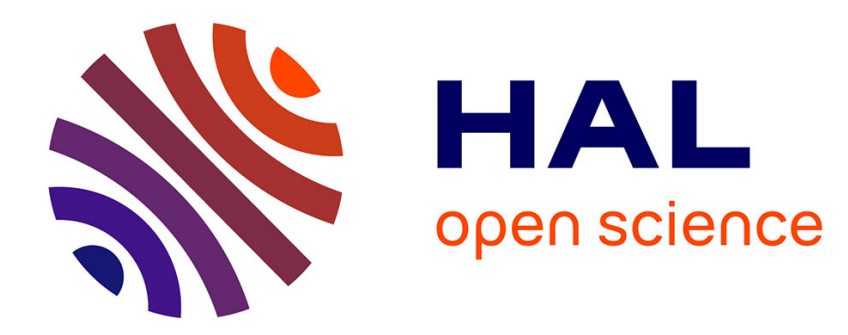

\title{
Les coordinations en vieux russe et le problème du Slovo o polku Igoreve \\ Robert Roudet
}

\section{To cite this version:}

Robert Roudet. Les coordinations en vieux russe et le problème du Slovo o polku Igoreve. ELADSILDA, 2020, Syntaxe des langues slaves: de la norme à la transgression, 4. hal-02873343

\section{HAL Id: hal-02873343 \\ https://univ-lyon3.hal.science/hal-02873343}

Submitted on 18 Jun 2020

HAL is a multi-disciplinary open access archive for the deposit and dissemination of scientific research documents, whether they are published or not. The documents may come from teaching and research institutions in France or abroad, or from public or private research centers.
L'archive ouverte pluridisciplinaire HAL, est destinée au dépôt et à la diffusion de documents scientifiques de niveau recherche, publiés ou non, émanant des établissements d'enseignement et de recherche français ou étrangers, des laboratoires publics ou privés. 


\title{
Les coordinations en vieux russe et le problème du Slovo o polku Igoreve
}

\author{
Robert Roudet \\ Université Jean Moulin Lyon 3, CEL \\ rroudet@wanadoo.fr
}

Le but de ce travail est de donner un exemple frappant d'évolution de la norme écrite. Nous nous penchons sur l'évolution de la coordination, en partant de la norme vieux russe que nous comparons à la norme de la langue moderne. Il est très facile de voir un changement important : la norme vieux russe était une abondance de coordinations qui est totalement exclue en langue moderne, car non seulement elle déboucherait sur une très grande lourdeur, mais même sur des énoncés fautifs. Nous voyons ensuite l'importance que ceci a eu pour la discussion sur l'authenticité du Slovo o polku Igoreve : ce manuscrit se rapproche par son emploi très modéré des coordinations de la langue moderne, mais une brillante étude de A. Zaliznjak qui compare ce manuscrit avec celui de la Zadonščina permet de conclure que ceci est au contraire une preuve très forte pour l'authenticité.

Mots-clés : coordination, norme, langue écrite, Slovo o polku Igoreve, Zadonščina, Zaliznjak

Цель этой работы - дать особенно ясный пример изменения нормы письменного языка. Мы представляем здесь изменения нормы сочинительной связи, сопоставляя положение в древнерусском языке с положением в современном языке. Наблюдается очень большая разница: норма древнерусского языка допускает очень широкое употребление сочинительных конструкций, которое исключено в современном языке, ибо оно привело бы не только к неуклюжему тексту, но и порой к совершенно недопустимым конструкциям. Затем мы рассматриваем роль первостепенной важности, которую сыграл этот факт в споре о подлинности Слова о полку Игореве: эта рукопись очень умеренным употреблением сочинения близка к более поздним текстам, но блестящее исследование А. Зализняка сравнительным анализом Слова и Задонщины доказывает, что такое положение дел на самом деле является очень сильным аргументом в пользу подлинности.

Ключевые слова: сочинительная связь, норма, письменный язык, Слово о полку Игореве, Задонщина, Зализняк

The purpose of this essay is to give a striking example of the evolution of the norm of the written language. We shall consider the evolution of coordination, starting from the norm of Old Russian which we will compare with the norm of the modern language. It is very easy to see an important change. The norm in Old Russian consisted in an abundance of coordinations, which cannot exist in modern language as it would result not only in a considerable ponderousness but also in the production of an incorrect utterance. Then, we will consider the importance this has had for the question of the authenticity of Slovo o polku 
Igoreve: this manuscript comes close to the modern language norm by its very moderate use of coordination, but a brilliant essay by A. Zaliznjak, who compares this manuscript with the one of Zadonšcina, argues that it is, on the contrary, a very strong proof of its authenticity.

Keywords: coordination, norm, written language, Slovo o polku Igoreve, Zadonščina, Zaliznjak

\section{Introduction}

Ce travail est basé sur deux types d'éléments : d'une part, la question des coordinations en vieux russe, et, d'autre part, l'étude faite par Andrej Zaliznjak de ce point dans le cadre du Slovo o polku Igoreve; comme il apparaît, la non-observance dans cette œuvre de la norme la plus habituelle en vieux russe n'est pas à interpréter comme il l'a souvent été fait. Les pages qui suivent ont donc une valeur essentiellement informative, car rien de très nouveau par rapport aux travaux de Zaliznjak n'y est exposé ; cependant on verra ici apparaître quelques questions touchant à ce qu'est une norme, qui peut recouvrir en fait des choses relativement diverses.

\section{Les coordinations en vieux russe et en russe moderne}

Examinons tout d'abord la façon dont se pose le problème des coordinations en vieux russe par rapport à leur emploi en langue moderne. On observe un double changement : tout d'abord, il est parfaitement évident, même pour un non-spécialiste de langue ancienne, que les coordinations sont plus nombreuses en vieux russe qu'en langue moderne ; de surcroît, elles se trouvent parfois dans des positions où elles ne sont plus envisageables en langue moderne.

Nous envisagerons ici deux conjonctions $i$ et $a$. Donnons tout d'abord quelques exemples de cas où la différence par rapport au russe moderne est une simple question de fréquence; les exemples suivants sont tous tirés de la Chronique de Nestor ${ }^{1}$, il s'agit donc, en principe, d'une langue reflétant la norme du $X I I^{e}$ siècle :

Изъгнаша варяги за море и не даша имъ дани и почаша сами въ собп володпти. И не бъ въ нихъ правды, и въста родь на родъ, и быша въ нихъ усобицп, и воевати почаша сами на ся. И рпша сами в себп

Рече Володимиръ: «Кака есть въра ваша?» Они же рпша: «Впруемъ Богу, а Бохъмитъ ны учить, глаголя: обрпзати уды тайныя, а свинины не псти, а вина не пити, и по смерти съ женами похоть творити блудную»

1 Nous avons utilisé l'édition de cette chronique donnée dans Библиотека литературы древней Руси [2000], qui n'a guère de valeur scientifique, mais qui est suffisante pour le but que nous nous sommes fixé. 
Ceci est vrai également dans le discours direct (rapporté évidemment) :

Дивно видпхъ землю словеньску, идущю ми спмо. Видпхъ банп древяны, и пережьгуть я велми, и съвлекутся, и будуть нази, и обольются мытелью, и возмуть впникы, и начнуть хвостатися, и того собп добьють, одва вылпзуть еле живы, и обольются водою студеною, и тако оживут.

Mais on retrouve la même tendance plus tardivement. Citons un exemple de ceci (Vie de Dracula, $x v^{e}$ siècle) :

Царь же велми разсердити себе о том, и поиде воинством на него, и прииде на него со многими силами. Он же, собравъ елико импаше у себе войска, и удари на турковъ нощию, и множьство изби их. И не возможе противу великого войска малыми людьми и възратися.

Il est déjà parfaitement clair que la norme moderne ne pourrait tolérer une telle fréquence de coordinations.

Il convient maintenant de mentionner le second cas, celui où les coordinations se trouvent dans des positions où elles sont tout simplement impossibles en langue moderne ; on trouve fréquemment en vieux russe des coordinations entre une forme de participe (parfois en fonction gérondivale et jouant, il est vrai, souvent un rôle prédicatif) et le prédicat principal à une forme personnelle; exemples tirés des Chroniques :

Въставъ и рече: ... (он встал и сказал)

Посла послы и глаголя:

Жены русскія въсплакашася а ркучи: (жены русские заплакали, говоря...)

Cette dernière forme а ркучи a par la suite été parfois mal interprétée (sans doute par le fait de son étrangeté au vu d'une norme plus moderne) : on y a parfois vu, non pas une conjonction $a$ et la forme de participe présent actif nominatif féminin singulier (le masculin était река), mais une forme аркучи où le $a$ initial serait apparu pour des raisons phonétiques. Voici un autre exemple, tiré de La Vie de sainte Févronie, où I'on trouve une coordination reliant une proposition principale à un datif absolu, qui jouait le rôle d'une circonstancielle :

Пловущимъ же имъ по рпцп въ судпхъ, и нпкий человпкъ бп у блаженныя кнеини Февронии въ суднп, егоже и жена въ томъ же суднп бысть.

On peut donner encore un exemple plus tardif tiré de La Vie de Dracula :

Приидоша к нему нъкогда от турьскаго поклисарие и, егда внидоша к нему и поклонишась по своему обычаю, а капъ своих з главъ не сняша. 
On voit donc que ce phénomène de surabondance de coordinations est assez général. Nous n'aborderons pas une question qui pourrait après tout être posée : et si ces lexèmes $u$ et $a$ n'avaient pas la même valeur qu'en langue moderne et n'étaient pas sentis comme des coordinations, mais, par exemple, comme des particules ayant un rôle tout autre ? Cette question n'a pas d'importance pour la suite, le tout étant de voir que ces $u$ / $a$ se trouvent avec une fréquence qui frapperait même un lecteur peu attentif. Une question qui n'a pas été évoquée non plus pour l'instant mais que I'on verra apparaître par la suite est la suivante : dans quelle mesure ces emplois sont-ils une norme contraignante, c'est-à-dire peut-on envisager qu'un auteur de l'époque écrive autrement ? Nous pouvons dire déjà ceci : nous avons cité des exemples particulièrement frappants de ce point de vue, mais même si la tendance est manifeste, si l'on prend un auteur tel que Cyrille de Turov, on verra que la proportion de coordination, plus élevée qu'en langue moderne, sans doute, n'atteint malgré tout pas les proportions observées ici. Il ne s'agit donc pas d'une règle normative absolue, loin de là.

\section{Les coordinations et le Slovo o polku Igoreve}

Cependant certains spécialistes qui se sont penchés sur le manuscrit du Slovo o polku Igoreve (considéré en principe comme datant du $X I I^{e}$ siècle) ont manifesté leur surprise de voir que cette œuvre ne se pliait pas du tout à cette tendance générale et que, de ce point de vue, l'impression qui se dégageait de ce pilier de la littérature vieux russe était curieusement moderne; on ne peut que leur donner raison au vu de l'exemple suivant :

Другаго дни велми рано кровавыя зори свптъ повпдают. Чръныя тучи съ моря идутъ, хотятъ прикрыти 4 солнца, а в нихъ трепещуть синіи молніи. Быти грому великому, идти дождю стрплами съ Дону Великаго! Ту ся копіемъ, приламати, ту ся саблямъ потручати о шеломы Половецкыя, на рпцп на Каяль, у Дону Великаго.

Ce point pourrait sembler secondaire, sembler être une curiosité sans grande importance si ceci n'entrait pas dans une problématique qui a été au centre d'une discussion acharnée entre les philologues tenant de l'hypothèse que le Slovo était bien ce pour quoi il se donnait, c'est-à-dire un manuscrit authentique, et ceux qui supposait qu'il s'agissait d'un faux, comme il y en avait déjà eu plusieurs, des Chants ossianiques jusqu'aux faux manuscrits tchèques. On va voir que cette question des coordinations, soigneusement étudiée par Andrej Zaliznjak, a permis d'arriver d'une façon très originale à une conclusion inattendue. Notre but n'est pas du tout de retracer toute la querelle qui a opposé partisans et adversaires de l'authenticité du Slovo, mais un certain nombre d'éléments doivent tout de même être rappelés pour rendre compréhensible la signification de cet étrange écart entre cette œuvre et les textes vieux russes de la même époque sur l'emploi de ces coordinations. Rappelons donc les points essentiels. 
Le manuscrit du Slovo o polku Igoreve a été découvert en 1795, il a été édité et traduit par les collaborateurs de Musin-Puškin en 1800. Une copie en avait été faite auparavant pour l'impératrice Catherine II. Le manuscrit lui-même périt malheureusement dans l'incendie de Moscou en 1812 avec I'ensemble de la bibliothèque de Musin-Puškin. Le sujet du Slovo (I'expédition malheureuse du prince Igor contre les Polovtses) est mentionné dans la Chronique de Nestor en trois lignes environ à l'année 1185 et on a supposé au départ que l'auteur était plus ou moins contemporain de ces événements. Cependant, I'œuvre était surprenante, ne serait-ce que par son caractère unique dans la littérature de l'époque. Bien des passages semblaient de surcroît assez obscurs.

Un autre manuscrit, la Zadonščina, a été publié en 1852 qui relate la bataille, cette fois victorieuse pour les Russes, du Champ des Bécasses (Поле Куликово) en 1380. Les savants se sont querellés presque sur tout, mais un point fait l'unanimité : il y a un lien évident entre la Zadonščina et le Slovo, puisque des passages entiers de ce dernier manuscrit se retrouvent dans la Zadonščina. Précisons que le manuscrit de la Zandonščina a connu une histoire complexe, qu'il en existe six versions, dont une courte et on ne sait même pas avec certitude quelle est la version première.

La première réaction face à ce fait indiscutable que l'on retrouve dans la Zandonščina des passages entiers du Slovo a été de dire que c'était là une preuve que le manuscrit du prince Igor était donc authentique, puisqu'une $œ u v r e$ de la fin du xve siècle en avait emprunté des passages entiers. Pour plusieurs raisons, c'est I'hypothèse la plus raisonnable, ne serait-ce que parce que les extraits du Slovo sont disséminés à travers l'ensemble de la Zandonščina. Cependant, certains ont mis en doute cette vision des choses et ont soutenu que c'était très certainement l'inverse qui s'était produit, que le Slovo était un faux qui avait emprunté à la Zandonščina. C'est André Mazon qui a lancé cette querelle, qui jusque-là était latente, en publiant un travail (très maladroit dans sa forme et un peu trop impressionniste sur le fond, à notre avis) tendant à prouver que le Slovo était postérieur à la Zandonščina. Il s'attira les foudres de Jakobson qui publia en réponse un long travail, sensiblement mieux argumenté sur le plan linguistique mais détestable par l'agressivité moqueuse dont il fait preuve vis-à-vis de Mazon, prouvant exactement l'inverse. C'était là le début d'une longue querelle que nous ne pouvons même pas résumer ici, querelle à laquelle ont participé les russisants les plus divers, dont certains d'une compétence fort douteuse ; de surcroît, cette querelle avait toute une série d'implications idéologiques sans rapport avec la science. Un doute (doute relatif, car Jakobson avait été, dans son étude, malgré tout plus convaincant que Mazon) a donc plané un certain temps sur la question de l'authenticité du Slovo. 


\section{L'étude d'A. Zaliznjak}

Revenons-en maintenant à la question des coordinations en donnant un aperçu de la façon dont Andrej Zaliznjak a fait de ce point une preuve incontestable de l'authenticité du Slovo. Il a étudié simultanément cette œuvre et les différentes versions de la Zadonščina en établissant ce qu'il appelle un coefficient de juxtaposition (opposée à la coordination). Les textes qu'il étudie sont divisés en groupes prédicatifs (en gros, en propositions). Le coefficient de juxtaposition représente le pourcentage de propositions juxtaposées par rapport à l'ensemble. Pour que ceci soit significatif, il ne faut tenir compte que des propositions où le choix est possible : on élimine en particulier les propositions exclamatives et les propositions subordonnées avec conjonctions subordonnantes.

En comparant le texte du Slovo avec d'autres œuvres, cette méthode met effectivement en évidence une grande différence dans la fréquence des coordinations. Pour le Slovo, le coefficient de juxtaposition est de 66,4 \%, ce qui est proche de ce qu'on obtient avec un texte moderne, Metel', de Puškin, où ce coefficient est de $75 \%$ et avec un texte de Lermontov (Maksim Maksimyč) on arrive à $78 \%$. Les textes d'une époque proche du Slovo présentent, eux, un coefficient bien plus bas. Nous en donnons la liste cidessous :

- Владимир Мономах. Описание походов: 20 \%

- Ипатьевская летопись. Поход Игоря: 13 \%

- Сказание о Мамаевом побоище: 14 \%

- Хожение за три моря: 14,5 \%

A priori, ceci donnerait à penser que le Slovo n'est pas authentique.

Ensuite, A. Zaliznjak procède au découpage du Slovo et de la Zadonščina en trois types de passages : ceux communs aux deux manuscrits, ceux propres au Slovo et ceux propres à l'une des versions de la Zadonščina. Il établit le coefficient de juxtaposition pour ces différentes parties et nous donnons l'essentiel des résultats dans le tableau ci-dessous :

\begin{tabular}{|l|l|l|l|l|}
\hline $\begin{array}{l}\text { Слово о полку } \\
\text { Игореве }\end{array}$ & $\begin{array}{l}\text { Кирилло- } \\
\text { Белозерский } \\
\text { список }\end{array}$ & $\begin{array}{l}\text { Синодальный } \\
\text { список }\end{array}$ & $\begin{array}{l}\text { Синодальный } \\
\text { список }\end{array}$ & $\begin{array}{l}\text { Список } \\
\text { Ундольского }\end{array}$ \\
\hline $\begin{array}{l}67 \%(188 \text { cas } \\
\text { sur 281) }\end{array}$ & $62 \%$ & $54 \%$ & $50 \%$ & $35 \%$ \\
\hline $\begin{array}{l}65 \%(61 \text { cas sur } \\
94)\end{array}$ & $93 \%$ & $71 \%$ & $68 \%$ & $39 \%$ \\
\hline
\end{tabular}


Les chiffres de la première ligne correspondent aux pourcentages des parties indépendantes dans le Slovo et dans la Zadonščina, les chiffres de la seconde à ceux des parties communes.

À partir de là certaines constatations s'imposent. Tout d'abord le Slovo est homogène, le coefficient de juxtaposition est grosso modo le même dans les parties communes et les parties indépendantes. On peut en dire autant de la Zadonščina dans la variante de la copie Undol'skij, mais ce n'est pas là le cas le plus intéressant, car cette variante se rapproche tout simplement de la norme de l'époque pour l'ensemble. Au contraire les trois autres variantes présentent une chute très nette du coefficient de juxtaposition dans les parties indépendantes par rapport à ce qu'il est dans les parties communes. Il convient d'expliquer ceci.

Si I'on admet que le Slovo est authentique, I'explication est assez simple. Les auteurs de la Zadonščina, en empruntant des passages au Slovo, ont emprunté le style avec le contenu lui-même, puis, lorsqu'ils écrivaient par eux-mêmes, ils se rapprochaient (consciemment ou inconsciemment, peu importe) de la manière la plus courante d'écrire à leur époque. C'est là une explication toute naturelle.

Supposons maintenant que ce soit le Slovo qui ait fait des emprunts à la Zadonščina. Il faudrait alors admettre que I'auteur du Slovo ait emprunté une série de passages aux différentes variantes de la Zadonščina sur un critère formel, le peu de coordination (et pourquoi, la question ne peut guère avoir de réponse raisonnable). Ensuite, il aurait organisé ces passages en un ensemble cohérent en écrivant lui-même des passages en se tenant à cette particularité stylistique, un coefficient de juxtaposition assez élevé. On voit qu'on tombe ici dans des suppositions extravagantes.

\section{Conclusion}

Disons quelques mots en guise de conclusion. D'une part, il faut reconnaître que, s'agissant des coordinations, la norme n'était donc pas si absolue. Et reconnaissons enfin que l'étude d'A. Zaliznjak vient clore par des preuves irréfutables la discussion sur l'authenticité du Slovo. Ce que nous venons de citer n'est qu'une preuve parmi d'autres que I'on trouvera dans sa remarquable étude ; il est vrai que c'est là, à notre sens, peut-être la preuve la plus absolue qu'il donne. 


\section{Bibliographie}

Ермолай-Еразм, La Vie de sainte Févronie (Повесть о Петре и Февронии Муромских), in Лихачева Д.С., Дмитриева Л.А., Алексеева А.А. \& Понырко H.B. (eds.), 2000, Библиотека литературы Древней Руси, РАН. ИРЛИ, Saint-Pétersbourg: Наука, том 9.

Jakobson Roman, 1966, «La geste du Prince Igor », in Selected writings IV, La Haye, Paris : Mouton \& co, 106-132.

La chronique de Nestor (Повесть временных лет), in Лихачева Д.С., Дмитриева Л.А., Алексеева А.А. \& Понырко Н.B. (eds.), 2000, Библиотека литературы Древней Руси, РАН. ИРЛИ, Saint-Pétersbourg: Наука, том 1, 62-316.

La Vie de Dracula (Сказание о Дракуле), in Лихачева Д.С., Дмитриева Л.А., Алексеева А.A. \& Понырко Н.B. (eds.), 2000, Библиотека литературы Древней Руси, РАН. ИРЛИ, Saint-Pétersbourg: Наука, том 7, 460-472.

Le Dit du Prince Igor (Слово о полку Игореве), in Лихачева Д.С., Дмитриева Л.А., Алексеева А.А. \& Понырко Н.В. (eds.), 2000, Библиотека литературы Древней Руси, РАН. ИРЛИ, Saint-Pétersbourg: Наука, том 4, 254-268.

Mazon André, 1940, Le Slovo d'Igor, Paris : Droz (travaux publiés par I'I.E.S.), t. 20.

Roudet Robert, 2009, «La querelle du Dit du Prince Igor : les motivations socio-culturelles », in Beliakov Vladimir (éd.), La société russe à travers les faits de langue et les discours, Dijon : Éditions universitaires de Dijon, 87-98.

Roudet Robert, 2011, « Mazon et le Slovo d'Igor », Revue des Études Slaves, 92/1, 55-67.

Zaliznajk Andrej, 2004, Слово о полку Игореве: взгляд лингвиста, Moskva: Jazyki slavjanskoj kul'tury. 